\title{
1 Lagerungsmanagement und Lagerungstherapie in Anästhesie und Intensivmedizin
}

\author{
Klaus Lewandowski und Monika Lewandowski
}

Narkoseeinleitungsraum. Anlage einer Regionalanästhesie. Sitzend oder Seitenlage. Legen eines ZVK. Kopftieflage. Narkoseeinleitung. Rückenlage. Operationssaal. Umlagerung des Patienten auf den Operationstisch. Bauchlage, Seitenlage, Kopftieflage, etc., Intensivstation. Rückenlage, Oberkörperhochlage, Lagerungswechsel. Wann wird die Lagerung zur Herausforderung oder sogar zur Gefahr für den Patienten? Die Antwort lautet: Beim fettleibigen und besonders beim extrem fettleibigen Patienten!

Dieses Kapitel wird die Besonderheiten und Risiken der in Anästhesie und Intensivmedizin angewendeten Lagerungsformen erläutern und aufzeigen, dass ein optimiertes Lagerungsmanagement bestimmte respiratorische und kardiovaskuläre Beeinträchtigungen des Fettleibigen bessern und letztendlich sogar akute Erkrankungen günstig beeinflussen und Komplikationen vermeiden kann.

\subsection{Allgemeine Überlegungen}

Krankhaft Fettsüchtige vertragen körperliche Belastungen schlecht. Dies geht zurück auf die eingeschränkten respiratorischen und kardialen Reserven. Schon ein einfacher Lagerungswechsel, z.B. von der sitzenden in die liegende Position, kann an die Belastungsgrenzen von Atmung und Kreislauf stoßen. Dies kann je nach kardialer oder pulmonaler Vorschädigung des Patienten gefährlich werden.
Jeder Lagerungswechsel bei schwer übergewichtigen Patienten muss wohlüberlegt sein und schonend durchgeführt werden.

Eine optimale Lagerung kann entscheidend zur Entlastung von Lunge und Herz beitragen. Ist der Patient wach und ansprechbar, kann er dabei mitwirken, eine geeignete Lagerungsform zu finden. Die Lagerung, in der er sich wohlfühlt und gut durchatmen kann, kommt der idealen Lagerung oft sehr nahe. Doch gibt es durchaus Indikationen, in denen andere, für den Patienten u.U. ungewohnte oder ,unbequemere" Lagerungen von Vorteil sein können.

In der Anästhesie richtet sich die Auswahl der Lagerungsform nach den speziellen Erfordernissen des operativen Vorgehens und erlaubt nur wenig Variation. Hier muss die Lagerung des Fettleibigen besonders sorgfältig geplant werden. Chirurgen und Anästhesisten müssen hierbei eng zusammenarbeiten und rasch notwendig werdende intraoperative Lagerungswechsel bei Verschlechterung von kardiorespiratorischen Parametern des fettleibigen Patienten müssen von beiden Parteien schnell realisiert werden.

Die adäquate Lagerung von Fettsüchtigen ist ein Thema, das überraschenderweise bisher wissenschaftlich wenig untersucht wurde. Zu Unrecht, denn die korrekte Lagerung ist auch in der Anästhe- 

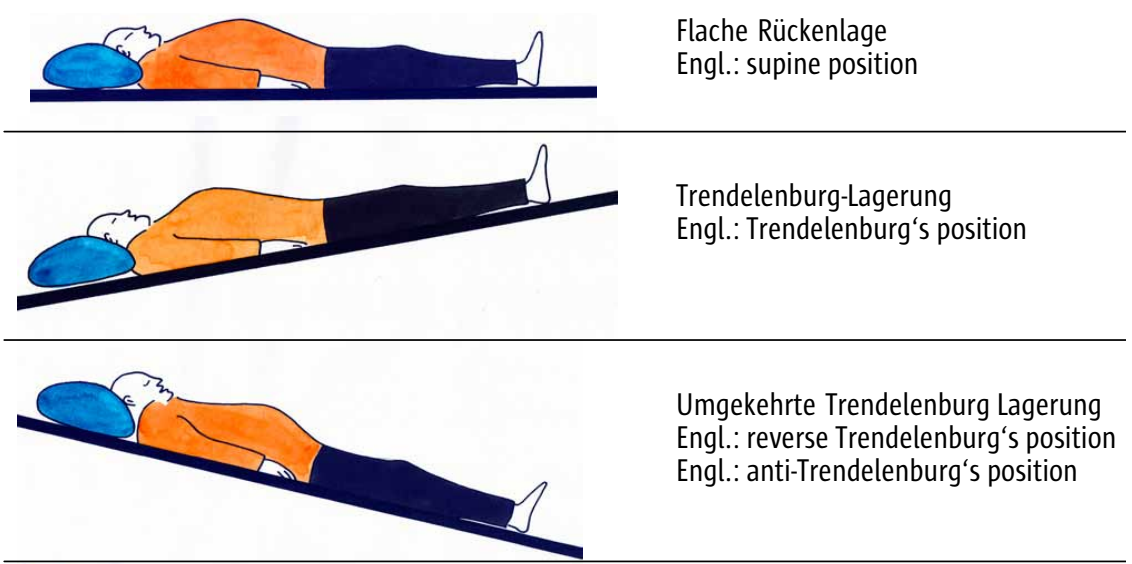

Umgekehrte Trendelenburg Lagerung Engl.: reverse Trendelenburg's position Engl.: anti-Trendelenburg's position
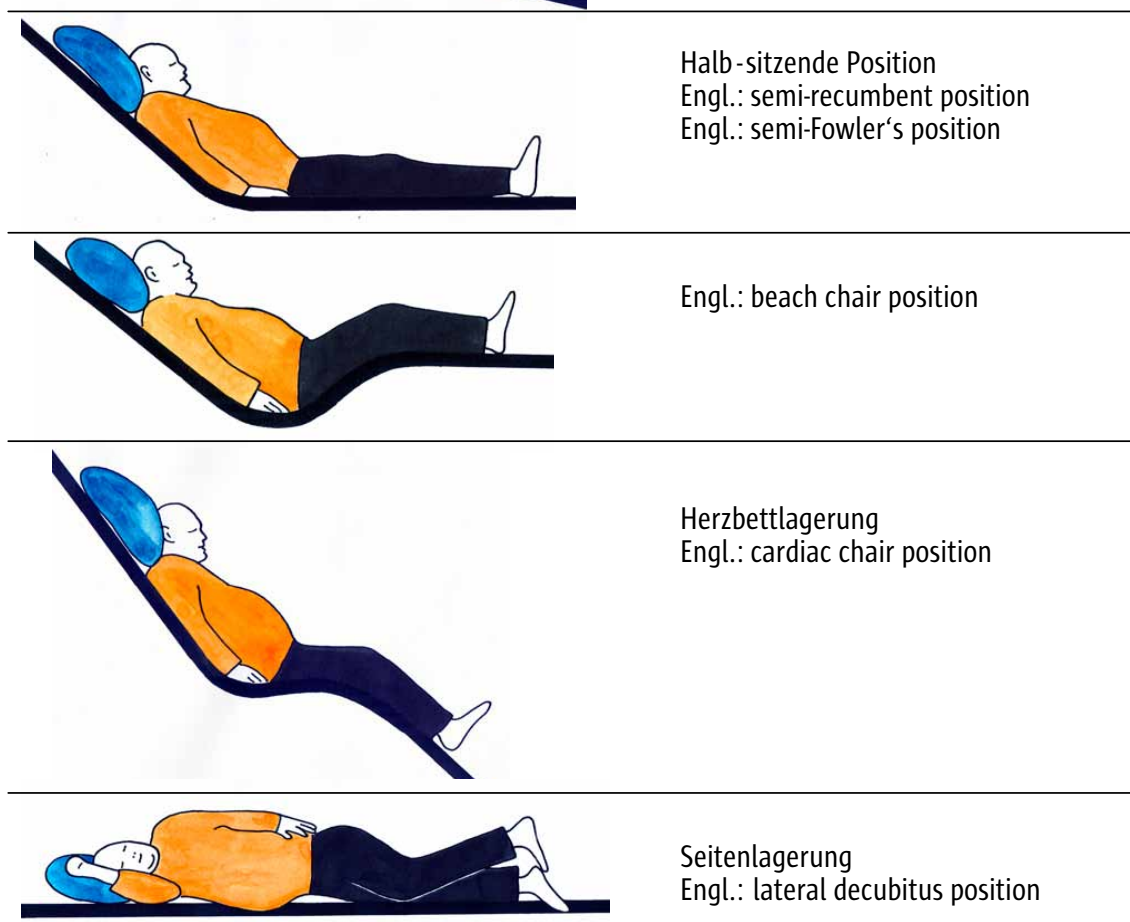

Seitenlagerung

Engl.: lateral decubitus position

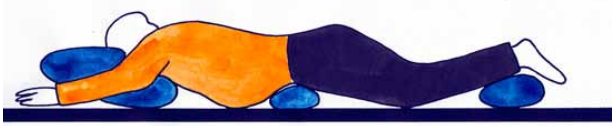

Bauchlage

Engl.: prone position

Abb. 1 Die verschiedenen Lagerungsformen im Überblick (aus Lewandowski u. Lewandowski 2009)

sie und Intensivmedizin von großer Bedeutung für den Patienten mit Adipositas per magna, damit sich der krankheitsgeschwächte Organismus optimal erholen kann. Abbildung 1 veranschaulicht synoptisch die verschiedenen, in Anästhesie und Intensivtherapie des Übergewichtigen verwendeten, Lagerungsformen, deren Vorteile und Risiken im Folgenden ausführlich vorgestellt werden. 


\subsection{Die sitzende Position}

Die Anlage einer Spinal- oder Epiduralanästhesie ist die klassische Indikation für eine sitzende Position, da es dem Patienten im Sitzen leichter fällt, den Körper optimal, d.h. mittig ohne Seitenneigung, vorzubeugen und die Punktion zu erleichtern. Gilt das auch für den fettleibigen Patienten?

Zunächst konnte gezeigt werden, dass die sitzende Position gegenüber der stehenden Position zu keinen wesentlichen Einschränkungen in der Lungenfunktion Übergewichtiger, gemessen an der forcierten Vitalkapazität (FVC) und dem forcierten Exspirationsvolumen in der 1. Sekunde $\left(\mathrm{FEV}_{1}\right)$, führt (Gudmundsson et al. 1997). Dennoch gibt es Hinweise von krankhaft übergewichtigen Schwangeren (BodyMass-Index [BMI] > 40), dass es günstiger ist, zur Anlage einer Epiduralanästhesie die Seitenlage einzunehmen. Es kam in der sitzenden Position in 18\% der Fälle zur akzidentellen venösen Kanülierung und in horizontaler Seitenlage nur bei 12\% der Schwangeren zur Punktion eines epiduralen Blutgefäßes. Die wenigsten Gefäßpunktionen fanden sich mit einer Häufigkeit von nur 5\% im Patientenkollektiv, dass zur Katheteranlage eine Seitenlagerung mit Kopftieflage einnahm (Chanimov et al. 2010).

Eine Kopftieflagerung des übergewichtigen Patienten, besonders des wachen spontan atmenden, halten die Autoren jedoch für sehr gefährlich und empfehlen diese nicht.

Eine horizontale Seitenlagerung ist jedoch, wie weiter unten ausgeführt, eine gute Alternative zur sitzenden Position.

Interessant zu wissen ist es, dass sich zum einen der Abstand zwischen Haut und Epiduralraum mit zunehmendem Gewicht und BMI des Patienten verlängert. Es überrascht jedoch die Tatsache, dass sich der Abstand auch verlängert, wenn die Punktion in Seitenlage statt in sitzender Position erfolgt (Hamza et al. 1995).

\subsection{Die flache Rückenlagerung}

\subsubsection{Der wache, spontan atmende Patient}

Legt sich ein normalgewichtiger Mensch vom Stehen oder Sitzen auf den Rücken, kommt es zu einem erhöhten venösen Rückfluss zum Herzen. Das Herzzeitvolumen, der pulmonale Blutfluss und der Blutdruck steigen. Auch die Lungenfunktionsparameter verschlechtern sich, allen voran nimmt die funktionelle Residualkapazität (FRC) deutlich ab. Das Abdo- men drückt von unten gegen das Zwerchfell und schränkt dessen Beweglichkeit ein. Tiefe Sedierung oder Anästhesie mit Muskelrelaxation können diese Effekte verstärken.

Der stark adipöse Patient toleriert diesen Lagerungswechsel deutlich schlechter. Schon der einfache Lagerungswechsel von der sitzenden in die liegende Position kann bei Fettleibigen an die Belastungsgrenzen von Atmung und Kreislauf stoßen:

- Das Herzzeitvolumen steigt um 35\% an.

- Der mittlere pulmonalarterielle Druck steigt um $31 \%$.

- Die Herzfrequenz fällt um $6 \%$.

- Die periphere Resistance reduziert sich um $21 \%$ (Paul et al. 1976).

- Der Sauerstoffverbrauch liegt um 11\% höher als im Sitzen.

Eine Studie aus dem Jahr 2005 untersuchte die Veränderungen der Lungenvolumina, wenn sich spontan atmende, stark übergewichtige Patienten mit einem BMI von $44 \pm 3$ aus der sitzenden Position in Rückenlage begaben. Schon im Sitzen hatten die übergewichtigen Patienten im Vergleich zu normalgewichtigen Personen Zeichen einer restriktiven Lungenfunktionseinschränkung, die sich in einer niedrigen totalen Lungenkapazität (TLC) und einer niedrigen FRC/TLC-Rate zeigten. Legten sich die Patienten auf den Rücken, fiel die TLC im Mittel um $80 \mathrm{ml}$ und die FRC um nur $70 \mathrm{ml}$. Bei gesunden Normalgewichtigen reduzierte sich die FRC im Mittel um $730 \mathrm{ml}$, wenn sich die Personen auf den Rücken legten. Messungen der respiratorischen Resistance ergaben doppelt so hohe Werte als bei Normalgewichtigen sowohl in sitzender Position als auch in Rückenlage (Watson u. Pride 2005).

Im Fall extremer Adipositas ist besondere Vorsicht bei der Rückenlagerung dieser Patienten geboten, ja man muss sogar empfehlen, diese, wenn möglich, nicht anzuwenden. Patienten mit massiver Adipositas haben oft seit Jahren nicht mehr in Rückenlage gelegen und sogar im Sitzen geschlafen.

Tsueda et al. berichteten über den Fall eines 38-jährigen Patienten mit einem BMI von 84. Der Patient war dyspnoeisch schon beim normalen Gehen, schlief seit Jahren nur noch sitzend. Die Lungenfunktionsuntersuchungen zeigten starke Abweichungen von den Normalwerten. Die Vitalkapazität betrug nur noch 31\%, die FRC 55\% und die $\mathrm{FEV}_{1}$ lag bei $83 \%$ des vorhergesagten Wertes. Der arterielle Sauerstoff-Partialdruck $\left(\mathrm{PaO}_{2}\right)$ unter Raumluft wurde mit $38 \mathrm{mmHg}$ und der arterielle Kohlendioxid-Partialdruck $\left(\mathrm{PaCO}_{2}\right)$ mit $59 \mathrm{mmHg}$ gemessen. Als der Patient sich im 
Rahmen einer präoperativen radiologischen Untersuchung auf den Rücken legte, kam es zu massiver Dyspnoe, Zyanose und Bewusstlosigkeit, der ein Atemstillstand mit konsekutivem Herzstillstand folgte. Eine Reanimation blieb erfolglos. Fälle wie dieser prägten den Begriff "obesity supine death syndrome“" (Tsueda et al. 1979).

\section{Die Rückenlage ist für den stark übergewichtigen Patienten gefährlich.}

\subsubsection{Der intubierte, beatmete und sedierte Patient}

Pelosi et al. (1999) verglichen pulmonalen Gasaustausch, Lungenvolumina, Elastance des respiratorischen Systems, Druck-Volumen-Kurven und den intraabdominellen Druck (IAP) von neun normalgewichtigen und neun fettleibigen Patienten. Die normalgewichtigen Patienten hatten einen mittleren BMI von 23,1 $\pm 1,2$, die Übergewichtigen kamen mit einem mittleren BMI von 51, $\mathrm{O} \pm 8,2$ vor allem zur Magenband-Operation. Vor der Operation zeigten die Lungenfunktionstests in der Gruppe der Übergewichtigen eine FVC von im Mittel 93\%, die FEV lag im Mittel bei $92 \%$ der vorhergesagten Werte und der Raumluft- $-\mathrm{PaO}_{2}$ bei $88 \pm 9 \mathrm{mmHg}$.

Unmittelbar nach dem abdominalchirurgischen Eingriff wurden an den noch anästhesierten, paralysierten und beatmeten Patienten auf der Intensivtherapiestation folgende pulmonale Parameter erhoben: Bei einem PEEP von o $\mathrm{CmH}_{2} \mathrm{O}$ und volumenkontrollierter, nach idealem Körpergewicht eingestellter Beatmung, fand man bei den Fettleibigen im Vergleich zu den Normalgewichtigen

- einen um etwa $100 \mathrm{mmHg}$ niedrigeren $\mathrm{PaO}_{2}$ bei einer $\mathrm{FiO}_{2}$ von 0 , 5 ,

- einen höheren $\mathrm{PaCO}_{2}$,

- ein deutlich geringeres endexspiratorisches Lungenvolumen,

- signifikant höhere Elastance-Werte für das respiratorische System, die Lunge und die Brustwand und

- einen etwa doppelt so hohen intraabdominellen Druck (IAP).

Eine Erhöhung des PEEP auf $10 \mathrm{cmH}_{2} \mathrm{O}$ verbesserte die respiratorische Funktion deutlich bei den Fettleibigen, bei den normalgewichtigen Patienten waren keine bemerkenswerten Veränderungen feststellbar. Alle Messungen wurden in Rückenlage durchgeführt (Pelosi et al. 1999).

\subsubsection{Besonderheiten beim Patienten mit massiver abdomineller Fettverteilung}

\begin{abstract}
Ein besonderer Fall von massiver Adipositas wurde 1981 beschrieben (Wyner et al. 1981). Eine 46-jährige Frau mit einem BMI von 122 (sic!), die seit längerem bettlägerig war, kam zur Pannikulektomie und Magenverkleinerung. Der abdominale Pannus reichte der Patientin bis zum Knie. Die Patientin litt an obstruktivem Schlaf-Apnoe-Syndrom (OSAS), Diabetes, arterieller Hypertension und koronarer Herzkrankheit. Sie tolerierte nur noch die sitzende Position. Der Raumluft- $\mathrm{PaO}_{2}$ lag bei $65 \mathrm{mmHg}$, der $\mathrm{PaCO}_{2}$ bei $40 \mathrm{mmHg}$. Zur Operation wurde eine Allgemeinanästhesie in Rückenlage eingeleitet, unter volumenkontrollierter maschineller Ventilation mit einem Tidalvolumen von $1600 \mathrm{ml}$ bei einer Beatmungsfrequenz von 12/Minute, einem Beatmungsspitzendruck von über $60 \mathrm{cmH}_{2} \mathrm{O}$ und einer $\mathrm{FiO}_{2}$ von 1,0 . Es wurde mit dieser Beatmungseinstellung ein $\mathrm{PaO}_{2}$ von $173 \mathrm{mmHg}$ erzielt. Intraoperativ wurde dann der abdominale Pannikulus senkrecht angehoben, der $\mathrm{PaO}_{2}$ verbesserte sich schlagartig auf $476 \mathrm{mmHg}$. Die Operation konnte erfolgreich beendet werden und die $\mathrm{Pa}$ tientin am 4. postoperativen Tag von der Beatmung entwöhnt werden. Allerdings verstarb die Patientin am 9. postoperativen Tag, vermutlich an den Folgen einer Endokarditis.
\end{abstract}

Auch wenn aus heutiger Sicht die Beatmungseinstellung mit ihren exzessiven Volumina und Drücken, die sich nicht am idealen Körpergewicht orientieren, als äußerst lungenschädigend anzusehen ist und keinesfalls mehr Anwendung finden sollte, so zeigt der Bericht doch eindrucksvoll, wie die extremen Fettmassen - besonders in Rückenlage - die pulmonale Situation beeinträchtigen.

So muss bei der Entscheidung für eine bestimmte Lagerung nicht nur dem allgemeinen Gewicht des Patienten Rechnung getragen werden, sondern auch der speziellen Verteilung der Fettmassen am Körper. Insbesondere bei starker Ausprägung von abdominalen Pannikuli oder intraabdominalen viszeralen Fettmassen muss davon ausgegangen werden, dass diese in Rückenlage Druck auf die Vena cava inferior ausüben. Ähnlich wie bei Schwangeren kann es hierbei zum Vena cava Kompressionssyndrom mit vermindertem venösem Rückstrom zum Herzen kommen. Dies kann - wie bei Schwangeren - durch Unterlegen eines großen Keilkissens oder leichter Seitenlagerung vermieden werden. Ist die Rückenlage jedoch notwendig, kann auch das Anheben eines sehr großen Pannikulus bei pulmonalen Problemen während Anästhesie oder Intensivmedizin das Abdomen vom Druck entlasten. 


\subsection{Die Kopftieflagerung}

Die Trendelenburg-Lagerung, d.h. die Kopftieflagerung (s. Abb. 1), ist für den Patienten mit Adipositas per magna äußerst gefährlich! Dennoch gibt es Indikationen, wo sie zur Anwendung kommen muss. Dies sind im Wesentlichen operative Eingriffe, die nur in Kopftieflage optimal ausgeführt werden können. Diese Indikationen implizieren, dass die Patienten zum Zeitpunkt der Trendelenburg-Lagerung intubiert, beatmet und kontinuierlich kreislaufüberwacht sind.

Die Kopftieflagerung ist eine erhebliche Belastung für das respiratorische und kardiovaskuläre System des Adipösen. Blut wird von den unteren Körperabschnitten zum Herzen auto-transfundiert und belastet das rechte Herz. Gleichzeitig pressen die abdominalen Fettmassen das Zwerchfell nach oben und komprimieren die Lunge, wo zusätzlich auch noch die Fettansammlungen des Oberkörpers auf der Lunge lasten. Die FRC fällt ab, Atelektasenbildung und Hypoxämien stellen sich nach kurzer Zeit ein.

Eine ältere Studie aus dem Jahr 1976 hat hierzu Daten geliefert. 64 übergewichtige Patienten, die eine Laparotomie erhielten, wurden zwei Gruppen zugeordnet. Bei Gruppe I (mittlerer BMI 50) wurde die gesamte Operation in Rückenlage durchgeführt, während Gruppe II (mittlerer BMI 52) in $15^{\circ}$ Kopftieflagerung operiert wurde. Bei Gruppe II kam es mit Etablierung der Trendelenburg-Position zu einem massiven Abfall des $\mathrm{PaO}_{2}$ von $123 \pm 33 \mathrm{mmHg}$ auf 79 $\pm 29 \mathrm{mmHg}$ bei einer $\mathrm{FiO}_{2}$ von 0,4 (Vaughan u. Wise 1976).

Diese Daten sind eindrucksvoll und zeigen uns die Risiken der Kopftieflagerung bei Übergewichtigen, doch es gibt auch Studienergebnisse, die keine spektakulären $\mathrm{PaO}_{2}$-Abfälle in Trendelenburg-Position zeigen: Sprung et al. (2003) untersuchten sechs normalgewichtige (BMI $21 \pm 3$ ) und sechs stark übergewichtige Patienten (BMI $48 \pm 5$ ), die intubiert und maschinell beatmet eine Laparoskopie erwarteten. Vor Etablierung des Pneumoperitoneums wurden diese von der Rückenlage in Trendelenburg-Position gelagert. Die arterielle Oxygenierung der Übergewichtigen war zwar vor der Umlagerung signifikant schlechter als die der Normalgewichtigen $\left(\mathrm{PaO}_{2} 172 \pm\right.$ $47 \mathrm{mmHg}$ vs. $260 \pm 21 \mathrm{mmHg}$ bei $\mathrm{FiO}_{2}=0,5$ ), doch verschlechterte sich diese überraschenderweise nach Kopftieflagerung nicht mehr weiter. Allerdings wird an der Studie kritisiert, dass die jeweiligen Lagerungs-Intervalle nur für kurze Zeit (fünf Minuten) aufrechterhalten wurden. Ebenfalls muss die sehr kleine Studiengruppe bemängelt werden.
Eine Untersuchung aus dem Jahr 2006 wiederum gemahnt uns an die Risiken der Trendelenburg-Lagerung. Es wurden 15 moderat Übergewichtige (BMI von 25-29,9) mit 15 Normalgewichtigen (BMI von 1824,9) im Hinblick auf Oxygenierung und Hämodynamik bei Lagerungswechsel untersucht. Nach Einleitung einer Allgemeinanästhesie und Kopftieflagerung der Patienten hatten die Übergewichtigen einen $\mathrm{PaO}_{2}$ von $164 \pm 51 \mathrm{mmHg}$ bei einer $\mathrm{FiO}_{2}$ von 0,5 , bei den Normalgewichtigen lag der $\mathrm{PaO}_{2}$ bei $235 \pm$ $27 \mathrm{mmHg}$. Leider wurde in der Studie nichts über die Oxygenierung vor der Trendelenburg-Lagerung berichtet. Die hämodynamischen Parameter Herzfrequenz, arterieller Blutdruck und zentraler Venendruck waren in beiden Gruppen gleich. Diese Ergebnisse belegen, dass schon bei Patienten mit einem moderaten Übergewicht die Kopftieflagerung mit zusätzlichen Risiken verbunden ist. Dabei steht die Hypoxämie im Vordergrund (Meininger et al. 2006).

\section{Die Kopftieflagerung ist für den stark übergewichti- gen Patienten äußerst gefährlich.}

Die Kopftieflagerung ist für den Übergewichtigen, und insbesondere den stark Fettleibigen, die riskanteste Lagerungsform. Eine Kopftieflagerung des wachen adipösen Patienten ist keinesfalls zu empfehlen. Für eine Trendelenburg-Lagerung sollte der übergewichtige Patient immer intubiert und maschinell beatmet sein und unter kontinuierlicher Kreislaufüberwachung stehen. Die Kopftieflagerung sollte nur so kurz wie möglich durchgeführt und evtl. in geeigneten Momenten auch während einer Operation kurz aufgehoben werden, um ein pulmonales Rekruitmentmanöver durchzuführen. Bei Zeichen einer schweren Hypoxämie oder Kreislaufdepression ist die Kopftieflagerung sofort zu beenden. Für anästhesiologische und intensivmedizinische Manöver wie z.B. die Anlage zentralvenöser Katheter etc. sollte das Risiko einer Kopftieflagerung nicht eingegangen werden.

\section{Keine Kopftieflagerung bei Fettleibigen für die Anla- ge zentralvenöser Katheter.}

\section{Dislokation des Endotrachealtubus durch Trendelenburg-Lagerung}

Bisher ist man davon ausgegangen, dass Tubusdislokationen hauptsächlich ein Problem von großen 
Lagerungswechseln, beispielweise von der Rückenin die Bauchlagerung sind. In einer randomisierten kontrollierten Studie an 6o Übergewichtigen mit einem BMI von 35-6o konnte gezeigt werden, dass bei laparoskopischen Operationen sowohl die Gasinsufflation als auch die Umlagerung von der Rückenlage in $10^{\circ}$ Trendelenburg-Lagerung bei 15 der $30 \mathrm{~Pa}-$ tienten der Studiengruppe (50\%) zu Tubusdislokationen führte (meist tiefer in den rechten Hauptbronchus). In der Kontrollgruppe, die eine offene Laparotomie erhielten, waren es nur sechs der zo Patienten (20\%) (Ezri et al. 2003). Leider wurde in der Studie keine normalgewichtige Kontrollgruppe untersucht. Allerdings weisen die vorgelegten Studienergebnisse darauf hin, dass es auch schon bei geringen Umlagerungen von intubierten und maschinell beatmeten Übergewichtigen zu Tubusdislokationen kommen kann. Es ist daher wichtig, die korrekte Lage des Endotrachealtubus nach jeder Umlagerung zu verifizieren.

\subsection{Die Oberkörperhochlagerung}

Vergegenwärtigt man sich die pathophysiologischen pulmonalen und hämodynamischen Veränderungen bei Patienten mit ausgeprägten Formen der Adipositas, kann man annehmen, dass die verschiedenen Arten der Oberkörperhochlagerung (s. Abb. 1) für den Patienten während operativer Eingriffe und in der Rekonvaleszenz auf der Intensivtherapiestation von Vorteil sind. In diesen Positionen wird das Zwerchfell vom abdominellen Druck entlastet und die Atmung und Lungenausdehnung erleichtert.

\section{Die Oberkörperhochlagerung ist während Anästhesie} und Intensivtherapie für Übergewichtige von Vorteil.

\subsubsection{Die umgekehrte Trendelenburg-Lagerung}

Die umgekehrte Trendelenburg-Lagerung scheint die effektivste Lagerungsform unter den Oberkörperhochlagerungen zu sein, um den Gasaustausch beim stark übergewichtigen Patienten günstig zu beeinflussen. In einer randomisierten Studie wurden 26 schwer übergewichtige Patienten (BMI $56 \pm 3$ ) drei Lagerungsformen zugeordnet.

- Gruppe 1: $30^{\circ}$ umgekehrte TrendelenburgLagerung,

- Gruppe 2: flache Rückenlagerung und

- Gruppe 3: $30^{\circ}$ halbsitzende Position.
Die Patienten wurden intubiert, anästhesiert und mit 50\% $\mathrm{O}_{2}$ beatmet, dann wurde pulsoxymetrisch die sichere Apnoe-Zeit (SAP, safe apnea period) gemessen, d.h. wie lange es dauerte, bis die Sauerstoffsättigung von 100\% auf $92 \%$ abfiel. Die längste SAP fand sich in der Cruppe mit $30^{\circ}$ umgekehrter Trendelenburg-Lagerung (178 \pm 55 s). In Rückenlage lag die SAP nur bei $123 \pm 24 \mathrm{~s}$ (Boyce et al. 2003).

Perilli et al. (2000; 2003) untersuchten den Effekt der umgekehrten Trendelenburg-Lagerung auf Gasaustausch und Lungenmechanik bei krankhaft übergewichtigen Patienten, die sich bariatrischer Chirurgie unterzogen. Sie stellten fest, dass die umgekehrte Trendelenburg-Lagerung die geeignete Lagerungsform für die obere Abdominalchirurgie im Hinblick auf Blutdruckveränderungen und pulmonalen Gasaustausch darstellt. Auch in einer Studie zum Weaning von maschineller Ventilation, erwies sich die $45^{\circ}$ umgekehrte Trendelenburg-Lagerung als optimale Lagerung, bei der es zur signifikanten Steigerung des Atemzugvolumens bei abnehmender Atemfrequenz kam (Burns et al. 1994).

Die umgekehrte Trendelenburg Lagerung ist geeignet, den Gasaustausch besonders des stark Übergewichtigen während Anästhesie und Intensivtherapie zu verbessern.

\subsubsection{Beach-Chair-Lagerung}

Ganz aktuell hat die sog. Beach-Chair-Lagerung (s. Abb. 1) für Patienten mit Adipositas Beachtung gefunden. Der Patient wird $30^{\circ}$ Oberkörper hoch gelagert und die Beine werden zum Abdomen hin angezogen.

Valenza et al. (2007) untersuchten präoperativ 20 intubierte, muskelrelaxierte und maschinell beatmete übergewichtige Patienten (BMI von $42 \pm 5$ ) in Rückenlage und Beach-Chair-Lagerung. In Rückenlage war der IAP erhöht, die endexspiratorischen Lungenvolumina kleiner als normal, die Elastance der Lunge und Thoraxwand war erhöht und die Resistance des respiratorischen Systems ebenfalls höher. Mit der Umlagerung in die Beach-Chair-Position vergrößerte sich das Lungenvolumen und die Elastance der Lunge und Thoraxwand erniedrigte sich. Die Resistance veränderte sich nicht. Der $\mathrm{PaO}_{2}$ verbesserte sich um etwa $25 \mathrm{mmHg}$ bei einer $\mathrm{FiO}_{2}$ von o,6. Der Blutdruck fiel leicht ab, die Herzfrequenz änderte sich nicht. Der IAP, gemessen als Harnblasendruck, erhöhte sich in Beach-Chair-Position signifikant von 
$18 \pm 5 \mathrm{cmH}_{2} \mathrm{O}$ auf $24 \pm 4 \mathrm{cmH}_{2} \mathrm{O}$. Die Autoren interpretieren den erhöhten Harnblasendruck mit dem schwerkraft-bedingten Herunterrutschen des Darms ins Becken, was eine Entlastung des Zwerchfells zur Folge hat. Intraoperativ, während des Pneumoperitoneums zur Anlage eines Magenbandes, konnte nur die Kombination von Beach-Chair-Position und PEEP die Oxygenierung verbessern.

Insgesamt stellt uns die Studie eine vielversprechende Lagerungsform für den Übergewichtigen vor, die sowohl in Anästhesie als auch in der Intensivmedizin am intubierten und maschinell beatmeten Patienten eine Option darstellt.

Ob diese Lagerung auch für den wachen Patienten geeignet ist, wurde bisher nicht untersucht, erscheint aber aus pathophysiologischer Sicht sinnvoll.

\subsubsection{Die „Cardiac-Chair“-Lagerung}

Die „Cardiac-Chair“- oder Herzbettlagerung (s. Abb. 1) findet weltweit bei Normal- und Übergewichtigen mit Herzinsuffizienz und/oder Atemnot Anwendung. Es wird eine Position angestrebt, bei der die Rückenlehne auf ungefähr $40^{\circ}$ gekippt, im Bereich der Kniegelenke das Bett geknickt und das Fußende nach unten geklappt wird. Schließlich wird dann noch die ganze Liegefläche um etwa $45^{\circ}$ gekippt.

Viele Übergewichtige berichten spontan von einer Besserung der Atemnot, wenn sie von der Rückenlage oder einer anderen Position in die Herzbettlagerung gebracht werden. Dies ist über eine Verlagerung des IAP weg von der Lunge und einem verbesserten Einsatz der Atemhilfsmuskulatur zu erklären. Zusätzlich wird das Herz über einen gedrosselten venösen Rückstrom entlastet. Klinisch beobachtet man oft eine Verlangsamung der Atemfrequenz, eine Zunahme der Thoraxexkursionen und eine Normalisierung von Blutdruck und Herzfrequenz. In der arteriellen Blutgasanalyse lässt sich dann häufig eine Verbesserung der Oxygenierung und $\mathrm{CO}_{2}$-Elimination dokumentieren.

Leider liegen keine wissenschaftlichen Untersuchungen vor, in denen diese Beobachtungen in Maß und Zahl gefasst wurden. Dennoch scheint es aber aufgrund pathophysiologischer Überlegungen gerechtfertigt, diese Lagerungsform bei spontan atmenden und maschinell beatmeten übergewichtigen Patienten auf der Intensivtherapiestation einzusetzen.

Mit den verschiedenen Formen der Oberkörperhochlagerung wurde hier ein Lagerungskonzept vor- gestellt, das dem Übergewichtigen in Anästhesie und Intensivmedizin Vorteile bietet, wozu allerdings zurzeit nur wenige Studien vorliegen. Daher sollte man auch den wachen, spontan atmenden Patienten mit sehr ausgeprägtem Übergewicht in Oberkörperhochlagerung regelmäßig kardiopulmonal überwachen. Insbesondere wache Patienten bevorzugen die Oberkörperhochlagerung unter dem Bequemlichkeitsaspekt. Dabei sind die halbliegende Position und die Beach-Chair-Position beliebter als die umgekehrte $45^{\circ}$ Trendelenburg-Lagerung wegen des Gefühls des „nach unten aus dem Bett Rutschens“. Diesen Patientenwünschen kann sicher weitgehend nachgekommen werden, wenn nicht wichtige Gründe für die umgekehrte Trendelenburg-Lagerung sprechen. Kontraindikationen der Oberkörperhochlagerung sind in Tabelle 1 zusammengestellt.

\subsection{Die Seitenlage}

Die Seitenlagerung des Übergewichtigen (s. Abb. 1) wird zur Dekubitusprophylaxe häufig in der Intensivmedizin praktiziert, dennoch sind die Auswirkungen der Lagerung auf das respiratorische und kardiovaskuläre System des Adipösen wissenschaftlich kaum untersucht. Aus pathophysiologischen Überlegungen heraus muss man annehmen, dass die Seitenlagerung vom Übergewichtigen gut toleriert wird, da das Abdomen vom Pannikulus entlastet wird und damit der IAP sinkt und das Zwerchfell sich freier bewegen kann. Dies würde sowohl dem spontan atmenden, wachen Patienten als auch dem intubierten und beatmeten Patienten zugutekommen.

\subsubsection{Ventilation}

Überraschenderweise ändern sich bei wachen, spontan atmenden Übergewichtigen die Atemzugvolumina nicht mit der Art der Lagerung, wenn man die sitzende Position mit der Rückenlage und der Seitenlage vergleicht, obwohl die Volumina insgesamt gegenüber denen von Normalgewichtigen erniedrigt sind. Auch die Atemfrequenz ändert sich mit den Lagerungswechseln nicht. Dies ist bei Normalgewichtigen anders. Hier finden wir die größten Atemzugvolumina in Rückenlage und die niedrigsten in der Seitenlage, die Atemfrequenzen ändern sich bei Lagerungswechsel ebenfalls nicht (Pankow et al. 1998).

Bei normalgewichtigen Patienten trägt sowohl die rechte als auch die linke Lunge jeweils zur Hälfte zur Ventilation bei, wenn die Personen sitzen oder 
Tab. 1 Wichtigste Kontraindikationen für Oberkörperhochlagerung und Bauchlage

\begin{tabular}{|c|c|c|}
\hline & Oberkörperhochlagerung Kontraindikationen & Bauchlage Kontraindikationen \\
\hline \multirow[t]{6}{*}{ kardiovaskulär } & hämodynamische Instabilität & hämodynamische Instabilität \\
\hline & femoraler Katheter & vorangegangener Herzstillstand \\
\hline & extrakorporale Zirkulation & Blutungen \\
\hline & ventrikuläre Assist-Systeme & ventrikuläre Assist-Systeme \\
\hline & intraaortale Ballonpumpe & intraaortale Ballonpumpe \\
\hline & & nach kardiothorakalen Eingriffen \\
\hline \multirow[t]{4}{*}{ neurologisch } & ischämischer Hirninfarkt & Kopfverletzung (Rücksprache mit Neurochirurg erforderlich) \\
\hline & Rückenmarksverletzung & Rückenmarksverletzungen \\
\hline & & Hirndruckerhöhung (Rücksprache mit Neurochirurg erforderlich) \\
\hline & & Krampfanfälle \\
\hline \multirow[t]{3}{*}{ Kopf } & & hoher Augeninnendruck \\
\hline & & nach Gesichtschirurgie \\
\hline & & frische Tracheotomie $(<24 \mathrm{~h})$ \\
\hline \multirow[t]{5}{*}{ abdominal } & große Bauchwunde & nach Abdominalchirurgie \\
\hline & & offene Bauchwunde \\
\hline & & Peritonitis \\
\hline & & Pankreatitis \\
\hline & & gastraler Reflux \\
\hline \multirow[t]{4}{*}{ Skelett } & instabile Beckenfraktur & Kyphoskoliose \\
\hline & & Arthritis \\
\hline & & Polytrauma \\
\hline & & instabile Wirbelsäule \\
\hline sonstiges & & Schwangerschaft \\
\hline
\end{tabular}

auf dem Rücken liegen. In Rechts- und Linksseitenlage wird jeweils die abhängige, untenliegende Lunge besser ventiliert. Bei übergewichtigen Patienten ist das anders. Im Sitzen und in Rückenlage trägt die linke Lunge nur zu 43\% zur Ventilation bei. In Rechtsoder Linksseitenlage wird bevorzugt die obenliegende Lunge ventiliert. Die FRC ist in den SeitenlagePositionen in der untenliegenden Lunge verringert (Hurewitz et al. 1985).

Beim normalgewichtigen Patienten wird empfohlen, bei einseitiger Lungenerkrankung den Patienten auf die Seite der gesunden Lunge zu legen, da hier Lungenventilation und -perfusion in einem günstigen Verhältnis zu einander stehen (,Down with the good lung“ [Fishman 1981]), dieses Vorgehen muss bei Übergewichtigen evtl. überdacht werden, da die untenliegende Lunge im Vergleich zur obenliegenden weniger ventiliert wird. Dennoch kann hier keine Empfehlung gegeben werden, da keinerlei Studien zu diesem Vorgehen vorliegen.

\subsubsection{Atemarbeit}

In einer der wenigen Studien, in denen über Ergebnisse zur respiratorischen Funktion bei Übergewichtigen in Seitenlage berichtet wird, wurden acht $\mathrm{Pa}-$ tienten mit einem mittleren BMI von 43,9 $\pm 5,4$ evaluiert. Die Untersuchung konzentrierte sich auf die Messung des „air trapping“ und maß den intrinsi- 
schen PEEP (PEEPi) bei Normalgewichtigen und Übergewichtigen im Sitzen, in Rückenlage und in Rechtsseitenlage. Der PEEPi war bei den Übergewichtigen in jeder Lage deutlich gegenüber dem von Normalgewichtigen erhöht, jedoch war er in Rechtsseitenlage nur halb so hoch wie in Rückenlage (Pankow et al. 1998). Ein erhöhter PEEPi ist eine zusätzliche Last, welche die Atemmuskulatur in der Inspiration überwinden muss. In Rückenlage erhöht sich über diesen Mechanismus die Atemarbeit, während in Seitenlage deutlich weniger Atemarbeit anfällt.

Die Ergebnisse der vorliegenden Untersuchungen legen nahe, dass die Seitenlage im Vergleich zur Rückenlage günstiger für den übergewichtigen Patienten ist. Zur Dekubitusprophylaxe kann sich eine Seitenlagerung (eventuell auch mit erhöhtem Oberkörper) mit einer Oberkörperhochlagerung abwechseln. Eine flache Rückenlagerung ist zu vermeiden.

\section{Seitenlagerung kann bei Übergewichtigen den pul- monalen Gasaustausch verbessern.}

\subsubsection{Komplikationen der lang dauernden Seitenlagerung}

Die bei Seitenlage untenliegende Lunge ist bei Übergewichtigen einem hohen Druck ausgesetzt und wird weniger ventiliert, die FRC ist erniedrigt $(\mathrm{Hu}-$ rewitz et al. 1985). Besteht die Seitenlagerung über lange Zeit, können sich Probleme in der untenliegenden Lunge ergeben. Dies beschreibt eindrucksvoll der Fall eines 50-jährigen übergewichtigen $\mathrm{Pa}$ tienten, der in einer 4,5-stündigen Operation (radikale Nephrektomie) in Linksseitenlage operiert wurde. Der $\mathrm{PaO}_{2}$ nach 30 min OP-Dauer lag bei $138 \mathrm{mmHg}$ $\left(\mathrm{FiO}_{2} \approx 0,33\right)$. Am Ende der Operation war der $\mathrm{PaO}_{2}$ unter der gleichen Respiratoreinstellung auf $70 \mathrm{mmHg}$ abgefallen. Nach Extubation betrug der $\mathrm{PaO}_{2}$ mit Sauerstoffmaske $50 \mathrm{mmHg}$. Im Thoraxröntgenbild fanden sich ein einseitiges Lungenödem und Atelektasen der linken Lunge. Beatmung mit PEEP konnte innerhalb von 36 Stunden den Befund bessern (Baraka et al. 1987).

Dieser Fall soll daran erinnern, dass eine länger dauernde Seitenlage des Übergewichtigen eine Belastung für die untenliegende Lunge darstellt und die Lagerungsart auf der Intensivtherapiestation regelmäßig gewechselt werden sollte. Ein hämodynamisches und respiratorisches Monitoring sollte bei Patienten mit starkem Übergewicht auch in Seitenlage nicht vergessen werden.

\subsubsection{Dislokation epiduraler Katheter bei Umlagerung aus der Seitenlage}

Die Seitenlagerung hat ihre Berechtigung nicht ausschließlich in der Dekubitus-Prophylaxe. Die Anlage von epiduralen Kathetern in Anästhesie und Schmerztherapie spielt ebenfalls eine Rolle, die bei immobilen übergewichtigen Patienten meist in Seitenlage erfolgt. Der Lagerungswechsel von adipösen Patienten mit epiduralen Kathetern ist problematisch, da der Katheter von der Haut bis zum Epiduralraum über eine lange Strecke im Fettgewebe verläuft und in diesem Abschnitt den Bewegungen der Fettmassen beim Umlagern ausgesetzt ist. Dislokationen sind hier bei Umlagerung des Patienten leider häufig (Hamza et al. 1995; Hamilton et al. 1997).

Beim Umlagern des Übergewichtigen kann der Epiduralkatheter leicht dislozieren.

\subsection{Die Bauchlage}

\subsubsection{Operationen in Bauchlage}

Operationen in Bauchlage werden täglich in unseren Kliniken durchgeführt, auch bei übergewichtigen Patienten. Große Bedenken über die Risiken dieser Lagerungsform hat man als Anästhesist und Intensivmediziner besonders bei Patienten mit stärkerem Übergewicht. Die wissenschaftliche Literatur zu diesem Thema ist jedoch dürftig.

Noch 2010 ist die Lagerung eines krankhaft Übergewichtigen in Bauchlage für einen chirurgischen Wirbelsäuleneingriff einen Fallbericht in einem größeren Wissenschaftsjournal wert (Baxi u. Budhakar 2010). Die Autoren berichten über eine 45-jährige Frau, $161 \mathrm{~cm}$ groß und $122 \mathrm{~kg}$ schwer (BMI 47), die nur unter Atemnot auf dem Rücken liegen kann und unter OSAS, Hypertonus und Diabetes mellitus leidet. Nach endotrachealer Intubation und Bauchlagerung wurde ein $\mathrm{PaO}_{2} / \mathrm{FiO}_{2}$ von $170 \mathrm{mmHg}$ gemessen, die Sauerstoffsättigung lag bei $96 \%$. Der operative Eingriff dauerte 3,5 Stunden und war komplikationslos.

Der Fall zeigt, dass auch länger dauernde Eingriffe in Bauchlage bei stark übergewichtigen Patienten sicher ausgeführt werden können. Die Autoren mahnen dennoch zu besonders sorgfältiger Vorbereitung, um eventuellen Intubationsschwierigkeiten oder akzidenteller Extubation zu begegnen. Auch die Ri- 
siken von Augenschädigungen und Herzstillständen in Bauchlage dürfen nicht vernachlässigt werden.

Pelosi et al. (1996) konnten an einer kleinen Fallserie zeigen, dass Operationen in Bauchlage auch für übergewichtige Patienten sicher sind, und die Bauchlagerung sogar Lungenfunktion und Gasaustausch verbessern kann. Die Autoren untersuchten 1o endotracheal intubierte, anästhesierte und maschinell beatmete übergewichtige Patienten (BMI $34,6 \pm 4,8)$, die zu einer Elektivoperation in Bauchlage anstanden. Vor der Operation wurden alle respiratorischen Daten in Rückenlage erhoben und dann wurden nach 15-30 Minuten die Messungen in Bauchlagerung wiederholt. Bei der Bauchlagerung wurde darauf geachtet, dass eine freie Beweglichkeit des Abdomen durch untergelegte Schaumstoffkissen gewährleistet war. Das verabreichte Atemzugvolumen war in Rücken- und Bauchlage gleich. Die FRC verdoppelte sich nach Bauchlagerung und die Compliance der Lunge erhöhte sich. Der $\mathrm{PaO}_{2}$ verbesserte sich bei einer $\mathrm{FiO}_{2}$ von 0,4 signifikant von $130 \pm$ $31 \mathrm{mmHg}$ auf $181 \pm 28 \mathrm{mmHg}$ mit der Umlagerung in Bauchlage.

\subsubsection{Die Bauchlagerung in der Intensivmedizin}

Die Bauchlagerung (s. Abb. 1) kommt in der Intensivmedizin am intubierten und beatmeten Patienten zum Einsatz, wenn im Rahmen einer schweren Lungenerkrankung eine ausgeprägte Hypoxämie auftritt, die sich nicht durch andere Maßnahmen bessert. Die Beatmung in Bauchlage kann in dieser Situation den $\mathrm{PaO}_{2}$ im Vergleich zur Rückenlage deutlich steigern. Auf der Röntgenthoraxaufnahme und in der Computertomografie (CT) der Lunge lässt sich oft eine Auflösung der basalen pulmonalen Atelektasen feststellen. Gilt dies auch für den übergewichtigen Patienten?

Lediglich ein ausführlich dokumentierter Fall eines Patienten mit schwerem Übergewicht kann hierzu Erfahrungen liefern. Ein 56-jähriger Mann mit einem BMI von 42 wurde mit einem akuten respiratorischen Versagen auf eine Intensivtherapiestation eingeliefert. Am 2. Tag entwickelte sich ein akutes Lungenversagen („, acute respiratory distress syndrome“" ARDS), der Patient wurde intubiert, lungenprotektiv beatmet, sediert und paralysiert. Unter der $\mathrm{FiO}_{2}$ von 1,0 lagen der $\mathrm{PaO}_{2}$ bei $83 \mathrm{mmHg}$, der $\mathrm{PaCO}_{2}$ bei $59 \mathrm{mmHg}$ und die arterielle Sauerstoffsättigung bei $96 \%$. Am 7. Tag mit weiter persistierendem ARDS fanden sich in der Computertomografie bilaterale alveoläre Konsolidierungen in den posterioren Lungenabschnitten. Der Patient wurde in Bauchlagerung verbracht, mit Unterstützung des oberen Brustkorbs und des Beckens durch Schaumstoffpolster, sodass die freie Beweglichkeit des Abdomens garantiert war. Nach 12 Stunden Bauchlagerung kam es zu einem deutlichen Anstieg des $\mathrm{PaO}_{2} / \mathrm{FiO}_{2}$ von $93 \mathrm{mmHg}$ auf $137 \mathrm{mmHg}$, der $\mathrm{PaCO}_{2}$ fiel von $57 \mathrm{mmHg}$ auf $50 \mathrm{mmHg}$ ab und die arterielle Sauerstoffsättigung stieg von $93 \%$ auf $97 \%$. Der Patient wurde nun für 3 Tage je einmal täglich von der Rückenlage in die Bauchlage umgelagert, in der er für jeweils 12 Stunden verblieb. Am 19. Tag konnte der Patient erfolgreich von der maschinellen Ventilation entwöhnt werden. Er konnte nach einer Rehabilitationsphase am 60. Tag nach Hause entlassen werden (Chergui et al. 2007).

Dieser Fallbericht zeigt eindrucksvoll, dass auch der krankhaft übergewichtige Patient von der Beatmung in Bauchlage profitiert. Dennoch muss eine Beatmung in Bauchlage sowohl beim Normalgewichtigen als auch beim Übergewichtigen gut überlegt sein und kommt nurfür ausgewählte Fälle von ARDS mit schwerer Hypoxämie infrage. Für leichtere Lungenerkrankungen können mit den anderen Lagerungsformen (umgekehrte Trendelenburg-Lagerung) durchaus auch gute Erfolge erzielt werden.

Zur Bauchlagerung sollte der Übergewichtige intubiert und beatmet sein und der Kreislauf kontinuierlich überwacht werden.

\subsubsection{Die korrekte Bauchlagerung}

Die Bauchlagerung ist beim Übergewichtigen, besonders beim stark Übergewichtigen eine Herausforderung. Nur die korrekte Ausführung der Bauchlagerung ist bei diesen Patienten die Grundvoraussetzung dafür, dass optimale Bedingungen für eine Verbesserung der Lungenfunktion geschaffen werden, aber auch Lagerungsschäden vermieden werden.

Die aktive Phase der Umlagerung vom Rücken auf den Bauch ist kritisch, und sie muss gut vorbereitet sein. Zunächst müssen alle Beatmungsschläuche, Infusionszuleitungen und Katheter überprüft werden und ggf. verlängert werden. Lagerungshilfen wie Kissen und Polster müssen in ausreichender Menge und Größe bereitliegen. Für die Umlagerung des stark adipösen Patienten reichen drei bis vier Helfer nicht aus, die man sonst zur Umlagerung des Normalgewichtigen benötigt. Der Personalbedarf muss sich zum einen am Gewicht des Patienten orientieren, andererseits dürfen sich die Helfer nicht 
gegenseitig behindern, und es darf nicht zu Unübersichtlichkeiten kommen, in deren Folge Katheter herausgezogen werden. Mit fünfbis sechs kräftigen Helfern sollte auch der stärker Übergewichtige gelagert werden können. Dabei muss ein Helfer allein darauf abgestellt sein, den Atemweg zu sichern. Starke Kopfbewegungen oder -drehungen müssen unbedingt vermieden werden, da es dabei zu Tubusdislokationen kommen kann. Ein weiterer Helfer sollte darauf achtgeben, dass alle Katheter in situ bleiben. Wurde die Umlagerung korrekt ausgeführt, sind oberer Thorax und Becken durch Polster so unterstützt, dass der Bauch frei nach unten hängt und kein Druck auf das Abdomen ausgeübt wird. Keinesfalls dürfen die Vena cava inferior oder die Femoralvenen komprimiert werden, da ansonsten der venöse Rückstrom zum Herzen behindert wird, was eine schwere Hypotension zur Folge haben kann. Die Lagerungspolster müssen dabei für stark übergewichtige Patienten durchaus schon $60 \mathrm{~cm}$ dick sein. Das Monitoring von Atmung und Kreislauf sollte jetzt rasch wieder angeschlossen werden. Eine Lagerung in Bauchlage länger als 12 Stunden sollte nicht erfolgen, da sich sonst das Risiko für Gesichtsödeme und Lagerungsschäden erhöht.

Wenn der Patient dann umgelagert ist, müssen die Auflagepunkte identifiziert werden, die später zu Druckstellen führen können. Die Lagerung des Kopfes ist - besonders bei Patienten mit Adipositas nicht einfach. Besonders Augen, Kinn und Nase müssen druckfrei bleiben, um Nekrosen zu verhindern. Doch auch Knie und Füße müssen von Druck entlastet werden. Kontraindikationen der Bauchlagerung sind in Tabelle 1 zusammengestellt.

\subsection{Wann wie lagern?}

Man ist nach der Darstellung der Lagerungsformen mit ihren Vorteilen und Risiken für den Übergewichtigen überrascht, wie groß doch der Einfluss der Lagerung auf die physiologischen Parameter ist. Eine falsche Lagerung beeinträchtigt die Sicherheit unseres Patienten; eine optimale Lagerung hingegen kann den Krankheitsverlauf günstig beeinflussen. Welche Lagerungsform im Einzelfall zu bevorzugen ist, hängt zum einen maßgeblich von der anästhesiologischen oder intensivmedizinischen Intervention ab, die geplant ist. Zum anderen muss aber auch der Körperfülle des Patienten Rechnung getragen werden. Mit zunehmendem Ausmaß der Adipositas wird die korrekte Lagerung immer wichtiger.
Extrem fettleibige Patienten sollten keinesfalls ohne vorher die hohen Risiken abgewogen zu habenin Rückenlage oder Kopftieflage gebracht werden, da schwere Nebenwirkungen, wenn nicht sogar ein Kreislaufstillstand die Folge sein können.

Die Anamnese des Patienten kann schon Hinweise geben, welche Lagerungsformen für den Patienten gefährlich sind. Befragt man fettleibige Patienten, wie sie sich zu Hause betten, berichten sie häufig, dass sie nicht gut flach liegen können und sich zur Nacht mehrere Kissen unter den Oberkörper legen. Bei flacher Lagerung bekommen sie Luftnot, Schwindelgefühl, Beengung und Angst. Die Patienten können nicht mehr richtig „durchatmen“. Extrem fettleibige Patienten können teilweise nur im Sitzen schlafen. Diesen Äußerungen sollte für das perioperative Lagerungsmanagement Aufmerksamkeit geschenkt werden.

Tabelle 2 stellt geeignete Lagerungsformen für die wichtigsten anästhesiologischen und intensivmedizinischen Herausforderungen beim fettleibigen Patienten im Überblick vor. Neben den klassischen, oben erläuterten Lagerungsformen gibt es noch Lagerungen für besondere Indikationen, denen man in Anästhesie und Intensivmedizin immer wieder begegnet. Diese werden im Folgenden beschrieben.

\subsection{Lagerung zur Intubation und Extubation}

\subsubsection{Intubation}

Man ist bisher davon ausgegangen, dass die Intubation von Übergewichtigen gegenüber der von Normalgewichtigen erschwert ist. In einer prospektiven Untersuchung an 82 stark übergewichtigen Patienten mit einem mittleren BMI von $42 \pm 6$ war die endotracheale Intubation in 15\% der Fälle erschwert. Bei den 124 Normalgewichtigen lag die Rate nur bei 6\% (Frat et al. 2008).

Ursächlich werden für die erschwerte Intubation die Fettmassen an Gesicht, Hals und Oberkörper verantwortlich gemacht, die eine korrekte Lagerung behindern. Allerdings können auch die pharyngealen Fettmassen die Sicht behindern. Es gibt jedoch Studien, die für Übergewichtige eine veränderte Lagerung zur Intubation vorschlagen, um die Sichtverhältnisse zu verbessern.

Brodsky et al. (2002) berichteten nach Untersuchung an 100 Patienten mit krankhaftem Übergewicht (mittlerer BMI 47,5), dass die Höhe des BMI 
Tab. 2 Lagerungsmanagement des stark Übergewichtigen in Anästhesie und Intensivmedizin

\begin{tabular}{|c|c|}
\hline $\begin{array}{l}\text { anästhesiologische oder intensivmedizinische } \\
\text { Herausforderung }\end{array}$ & $\begin{array}{l}\text { Vorschläge für eine geeignete Lagerungsform des stark übergewichtigen } \\
\text { Patienten }\end{array}$ \\
\hline Anlage von Spinal- und Epiduralanästhesie & $\begin{array}{l}\text { sitzende Position oder } \\
\text { Seitenlage }\end{array}$ \\
\hline $\begin{array}{l}\text { Anlage von Spinal- und Epiduralanästhesie } \\
\text { in der Geburtshilfe }\end{array}$ & Seitenlage \\
\hline $\begin{array}{l}\text { wach und spontan atmend im Operationssaal } \\
\text { mit Regionalanästhesie }\end{array}$ & $\begin{array}{l}\text { Oberkörperhochlagerung oder } \\
\text { Seitenlage (mit Oberkörperhochlagerung), der Patient soll sich möglichst } \\
\text { selbst lagern }\end{array}$ \\
\hline Präoxygenierung & umgekehrte Trendelenburg-Lagerung \\
\hline Intubation & „ramped position“ \\
\hline intubiert und beatmet während der Operation & $\begin{array}{l}\text { nach den chirurgischen Bedürfnissen, } \\
\text { möglichst Oberkörperhochlagerung, langdauernde Rückenlagerung und } \\
\text { Kopftieflagerung vermeiden }\end{array}$ \\
\hline intraoperative Hypoxämie, Rekruitmentmanöver & $\begin{array}{l}\text { Oberkörperhochlagerung, } \\
\text { bevorzugt umgekehrte Trendelenburg-Lagerung }\end{array}$ \\
\hline Extubation & Oberkörperhochlagerung \\
\hline mögliche Re-Intubation & Extubation in „ramped position“ \\
\hline postoperativ & Oberkörperhochlagerung \\
\hline $\begin{array}{l}\text { wach und spontan atmend auf der } \\
\text { Intensivtherapiestation }\end{array}$ & $\begin{array}{l}\text { Oberkörperhochlagerung oder } \\
\text { Seitenlage (mit Oberkörperhochlagerung), der Patient soll sich möglichst } \\
\text { selbst lagern }\end{array}$ \\
\hline Schlaf-Apnoe-Syndrom & $\begin{array}{l}\text { Oberkörperhochlagerung oder } \\
\text { Seitenlage }\end{array}$ \\
\hline Dekubitus-Prophylaxe & $\begin{array}{l}\text { wechselnde Seitenlagerung oder } \\
\text { kontinuierlicher Lagerungswechsel im Rotationsbett } \\
\text { (Maximalgewicht }<160 \mathrm{~kg} \text { beachten!) }\end{array}$ \\
\hline schweres ARDS mit lebensbedrohlicher Hypoxämie & Bauchlagerung im Wechsel mit Rückenlage oder Oberkörperhochlagerung \\
\hline maschinelle Beatmung/Weaning & umgekehrte Trendelenburg-Lagerung \\
\hline
\end{tabular}

nichts darüber aussagt, ob mit einer erschwerten Intubation zu rechnen ist. Nur bei 12 Patienten war die Intubation problematisch. Bei den problematischen Fällen war der Halsumfang signifikant größer als bei den unproblematischen Fällen (Median 50,5 cm vs. $46 \mathrm{~cm}$ ), ein Zusammenhang mit dem BMI wurde nicht gefunden.

Eins der wichtigsten Kriterien für eine erfolgreiche Intubation des krankhaft Übergewichtigen nach direkter Laryngoskopie ist die Lagerung des Patienten. Die optimale Intubationsposition ist erreicht, wenn Kopf, Oberkörper und Schultern deutlich über den
Brustkorb angehoben sind. Ideal ist die Lagerung, wenn der Meatus auditorius externus mit der Fossa jugularis sternalis auf einer imaginären horizontalen Linie liegen (s. Abb. 2). In dieser Position konnten 99 von 100 krankhaft übergewichtigen Patienten mittels direkter Laryngoskopie erfolgreich intubiert werden (Brodsky et al. 2003).

Die Überlegenheit der hier geschilderten sog. „ramped position“ zur Intubation von Übergewichtigen konnte in einer randomisierten kontrollierten Studie an 6o Patienten mit einem BMI von 40 oder höher bestätigt werden. Die Kontrollgruppe wurde in 


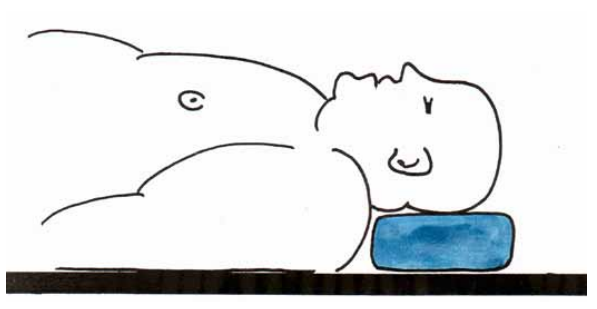

„sniff position“

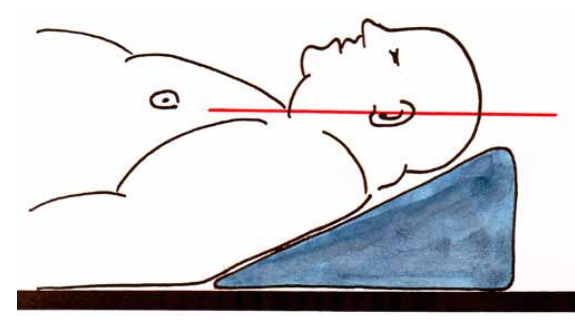

„ramped position“

Abb. 2 Die „ramped position“ sollte zur Intubation von Übergewichtigen bevorzugt werden. Bei der „ramped position“ wird der Oberkörper erhöht gelagert, sodass der Meatus auditorius externus mit der Fossa jugularis sternalis auf einer horizontalen Linie liegt. (aus Lewandowski u. Lewandowski 2009)

der „sniff position“ (,Schnüffelposition“ oder verbesserte Jackson-Position) gelagert, bei welcher der Kopf auf einem $7 \mathrm{~cm}$ hohen Polster gelagert wird. Die „ramped position" bot einen signifikant besseren Blick bei der direkten Laryngoskopie und wird für übergewichtige Patienten empfohlen (Collins et al. 2004).

Die „ramped position“ ist bei der endotrachealen Intubation des Übergewichtigen zu bevorzugen.

\subsubsection{Extubation}

Die Anzahl von Untersuchungen, die sich gezielt mit der endotrachealen Extubation bei Adipösen beschäftigen, ist sehr gering. Man stuft die Extubation des krankhaft Übergewichtigen gegenüber der Intubation allgemein als gefährlicher ein. In einer Studie an 82 Übergewichtigen mit einem mittleren BMI von $42 \pm 6 \mathrm{kam}$ es in $15 \%$ der Fälle zu einem Stridor nach Extubation (bei Normalgewichtigen nur in 3\% der Fälle) (Frat et al. 2008). Besonders Patienten mit einem OSAS sind von einer respiratorischen Obstruktionssymptomatik nach Extubation bedroht (Benumof 2004).

Eine Umfrage unter Anästhesisten in Großbritannien und Irland zeigt, dass von mehr als 40\% immer noch die Linksseitenlage oder Linksseitenlage kombiniert mit Kopftieflage (zur Verhinderung einer Aspiration) oder die Rückenlage bei der Extubation adipöser Patienten angewendet wird (Rassam et al. 2005). Zur Extubation sollte der Patient jedoch mit erhöhtem Oberkörper $\left(30-45^{\circ}\right)$ gelagert werden (Brodsky 2002).

\section{Zur Extubation sollte der übergewichtige Patient mit erhöhtem Oberkörper gelagert werden.}

In einer Arbeit zum Weaning von Übergewichtigen erwies sich die $45^{\circ}$ umgekehrte Trendelenburg-Lagerung als optimale Lagerung, bei der es zur signifikanten Steigerung des Atemzugvolumens bei abnehmender Atemfrequenz kam (Burns et al. 1994). Dass diese Lagerung auch zur Extubation bevorzugt werden könnte, liegt nahe. Studienergebnisse fehlen jedoch bisher.

\subsection{Veränderung der Lagerung bei intraoperativen Problemen}

Fettleibige Patienten sind intraoperativ besonders durch Atelektasen gefährdet. Insbesondere Rückenlagerung, Kopftieflagerung und ein Pneumoperitoneum begünstigen die Atelektasenbildung. In der Folge kann sich intraoperativ der pulmonale Gasaustausch deutlich, z.T. auch dramatisch, verschlechtern. In dieser Situation ist es sinnvoll und auch notwendig, der Atelektasenbildung entgegenzuwirken. Der Patient sollte unverzüglich - nach Rücksprache mit den Chirurgen - in die umgekehrte TrendelenburgLagerung gebracht werden und es sollte, falls sich der Gasaustausch nicht nachhaltig bessert, ein Rekrutierungs-Manöver durchgeführt werden. Hier hat sich die Kombination aus einem Vital-Kapazitätsmanöver mit einem anschließenden PEEP von $10 \mathrm{cmH}_{2} \mathrm{O}$ bewährt (Talab et al. 2009; Futier et al. 2010).

Extreme Rekrutierungsmanöver (insp. Druck $>40 \mathrm{mbar}$ ) können schwere kardiozirkulatorische Nebenwirkungen haben. 


\subsection{Postoperative Lagerung}

Doch auch postoperativ scheint die Oberkörperhochlagerung für den übergewichtigen Patienten vorteilhaft zu sein. Vaughan und Wise (1975) untersuchten 22 weibliche Patienten mit einem BMI von im Mittel 49 postoperativ nach Abdominalchirurgie. Sie entnahmen präoperativ und vom ersten bis zum dritten postoperativen Tag Blutgasanalysen bei Spontanatmung mit Raumluft. Der Patient wurde dabei in Rückenlage und in halbliegender Position untersucht. Präoperativ fand man keine signifikanten Unterschiede in der Oxygenierung in Rückenlage und halbliegender Position. An Tag 1 und 2 postoperativ schnitt die halbliegende Position besser ab. Am 3. postoperativen Tag wurden keine signifikanten Unterschiede zwischen Rückenlage und halbliegender Position mehr festgestellt.

Die vorgestellten Studien legen dem Intensivmediziner die Oberkörperhochlagerung für den übergewichtigen Patienten in der postoperativen Phase nahe. Man muss empfehlen, den Patienten schon unmittelbar postoperativ, also schon im Operationssaal mit dem Oberkörper hochzulagern und so zur Intensivtherapiestation zu transportieren.

\section{Der Übergewichtige sollte direkt postoperativ mit er-} höhtem Oberkörper gelagert werden.

\subsection{Lagerung bei Schlaf-Apnoe-Syndrom}

Übergewicht und Fettleibigkeit sind ein Risikofaktor für das OSAS. Lopez et al. (2008) evaluierten $290 \mathrm{~Pa}-$ tienten, bei denen ein gewichtsreduzierender operativer Eingriff durchgeführt werden sollte. Die Patienten wurden präoperativ auf das Vorliegen eines OSAS untersucht. Es litten 227 Patienten (78\%) daran. Die Patienten hatten einen mittleren BMI von 52 (Range 31-94). Es bestand eine positive Korrelation zwischen der Höhe des BMI und dem Auftreten von OSAS. Ebenfalls konnte ein Zusammenhang zwischen der Größe des Halsumfangs und OSAS bestätigt werden (Plywaczewski et al. 2008).

Der großen Häufigkeit von OSAS bei Übergewichtigen muss auch in der Intensivmedizin Rechnung getragen werden, sind doch die adipösen Patienten stärker als Normalgewichtige gerade in der Rekonvaleszenz nach chirurgischen Eingriffen von respiratorischen Problemen bedroht. Speziell beim lageabhängigen OSAS treten die Atmungsereignisse ausschließlich oder überwiegend in Rückenlage auf.
Diesen Patienten kann geholfen werden, indem die Rückenlage als Schlafposition vermieden wird (Verse et al. 2008). Als geeignetere Schlafpositionen werden eine aufrechte Schlafposition oder die Seitenlage beschrieben (Hakala et al. 200o; Itasaka et al. 2000).

Geeignete Schlafpositionen beim OSAS sind die Oberkörperhochlagerung und Seitenlage.

Dennoch ist das OSAS nicht in allen Fällen durch eine geänderte Lagerung während des Schlafs in den Criff zu bekommen. Je größer der BMI, desto geringer die Rate von Patienten, die z.B. auf die Seitenlage positiv reagieren (Itasaka et al. 20oo). Lässt sich das OSAS nicht durch die Lagerung beeinflussen, oder müssen bestimmte Lagerungsformen aus anderen Gründen eingehalten werden, wird man in diesen Fällen auf apparative Therapiemaßnahmen wie z.B. die CPAP („continuous positive airway pressure")-Atmung über eine Gesichtsmaske zurückgreifen müssen.

\subsection{Lagerung des beatmeten Patienten auf der Intensivtherapiestation}

Die Frage, welche Position für einen fettleibigen Patienten die beste ist, um eine optimale Zwerchfellexkursion während Beatmung und Weaning von der Beatmung zu ermöglichen, beschäftigt den Intensivmediziner schon lange. Studien zu diesem Thema gibt es kaum. Aus pathophysiologischer Sicht scheint es sinnvoll, Oberkörperhochlagerungen zu favorisieren, da sie das Zwerchfell vom intraabdominellen Druck entlasten und dessen freiere Beweglichkeit ermöglichen.

Um die optimale Weaning-Lagerung zu finden, wurden 19 intubierte, spontan atmende Patienten (CPAP oder "pressure support ventilation mode“) mit einer starken abdominellen Körpermasse, die entweder auf Übergewicht, Aszites oder abdominelle Distension zurückzuführen war, untersucht. Es wurden die Veränderungen von Atemzugvolumen und Atemfrequenz in Rückenlage $\left(\mathrm{o}^{\circ}\right)$, halbliegender Position $\left(45^{\circ}\right)$, sitzender Position $\left(90^{\circ}\right)$ und umgekehrter Trendelenburg-Position $\left(45^{\circ}\right)$ registriert. Die $45^{\circ}$ umgekehrte Trendelenburg-Lagerung erwies sich gegenüber der sitzenden Position als optimale Lagerung, bei der es zur signifikanten Steigerung des Atemzugvolumens bei abnehmender Atemfrequenz kam. Die halbliegende Position führte zu keiner Zunahme des Atem- 
zugvolumens, jedoch zu einem Rückgang der Atemfrequenz (Burns et al. 1994). Die Studie legt nahe, dass die umgekehrte Trendelenburg-Lagerung in der Weaningphase den anderen Lagerungsformen überlegen sein könnte.

Die $45^{\circ}$ umgekehrte Trendelenburg-Lagerung ist für das Weaning Übergewichtiger optimal.

Diese Studie zeigt auch, dass die halbliegende Position und die umgekehrte Trendelenburg-Position nicht ohne Weiteres gleichwertig sind. Man könnte hier den Aspekt diskutieren, ob bei der halbliegenden Position eine abdominelle Fettansammlung doch noch deutlich komprimiert wird, was den IAP mit den bekannten Auswirkungen auf die Zwerchfellexkursion wirksam steigert. Es scheint vertretbar zu sein, die umgekehrte Trendelenburg-Lagerung für die maschinelle Ventilation von Intensivpatienten mit hohem BMI zu empfehlen.

\subsection{Kontinuierlicher Lagerungswechsel im Rotations-Bett}

Spezielle motorgetriebene Bettsysteme erlauben einen kontinuierlichen Lagerungswechsel um die Längsachse des Patienten. Ist der seitliche Rotationswinkel größer als $40^{\circ}$ spricht man von einer kinetischen Therapie. Das System wurde entwickelt, um Schäden der Immobilisation wie Thrombosen oder Dekubitus zu mindern. Zudem soll die Lagerung im Rotationsbett den pulmonalen Gasaustausch durch Mobilisierung des tracheobronchialen Sekrets verbessern. Es konnte mit der Behandlung im kinetischen Bett die Prävalenz von Pneumonien bei maschinell beatmeten Intensivpatienten gesenkt werden (Bein 1998; Goldhill et al. 2007).

Goldhill et al. (2007) haben 2007 eine Metaanalyse vorgelegt, die untersucht, ob Rotationsbetten repiratorische Komplikationen verhindern oder erfolgreich therapieren können. Die Autoren konnten im Zeitraum von 1987 bis 2004 insgesamt 16 randomisierte kontrollierte Studien identifizieren, die den Effekt einer Rotationstherapie im Vergleich zu einer manuellen Lagerungstherapie untersucht haben. Die Meta-Analyse ergab, dass eine Rotationstherapie die Prävalenz der Pneumonie senkt, aber keinen Effekt auf die Dauer einer maschinellen Ventilation oder die Behandlungsdauer auf der Intensivtherapiestation hat. Die Autoren stellen fest, dass es nur wenige Hinweise gibt, welche Parameter einen Erfolg der Rotationstherapie wahrscheinlich machen. Dabei scheinen die Einstellung der Rotationsparameter wie Winkel, Frequenz, Dauer, Pausen oder zusätzliche Bedingungen wie Vibration, Perkussion oder Pulsation eine Rolle zu spielen. Möglicherweise hat die Art der Erkrankung, sowie Körpergröße und -gewicht ebenfalls einen Einfluss, wenngleich es bisher dazu keine Daten gibt. Die Autoren spekulieren, dass besonders die Gruppe der Patienten mit hohem BMI von der Rotationstherapie profitiert, da diese Patienten während ihres Aufenthaltes auf der Intensivtherapiestation überproportional von respiratorischen Problemen und Komplikationen bedroht sind. Zudem werden diese Patienten möglicherweise im intensivmedizinischen Alltag wegen des hohen zeitlichen und personellen Aufwands seltener manuell umgelagert als Normalgewichtige. Doch sind dies alles nur Aspekte, die diskutiert werden können, da es zurzeit keine Daten gibt, welche diese Hypothesen stützen.

\section{Zusammenfassung}

Ein konsequentes Lagerungsmanagement in Anästhesie und Intensivmedizin ist sehr wichtig, besonders für den fettleibigen und extrem fettleibigen Patienten. In der Anästhesie kann die Lagerung den Grundstein zu einer sicheren Narkose legen, eine falsche Lagerung aber auch den Patienten gefährden. Speziell Kopftieflagerungen oder langdauernde Rückenlagerungen können zu erheblichen intraoperativen und postoperativen pulmonalen Problemen führen.

In der Intensivmedizin ist die Lagerung eine effektive medizinische Maßnahme, die den Krankheitsverlauf günstig beeinflussen kann. Wichtig ist aber besonders, dass auch hier eine falsche Lagerungstherapie erheblichen Schaden anrichten kann. So wurden Todesfälle durch falsche Lagerung beschrieben. Die Oberkörperhochlagerung ist für den wachen, ansprechbaren adipösen Patienten geeignet und kann sich mit der Seitenlagerung (eventuell auch mit erhöhtem Oberkörper) zur Dekubitusprophylaxe abwechseln. Flache Rückenlagerung ist zu vermeiden und die Trendelenburg-Position ist für den nicht intubierten und beatmeten Patienten gefährlich. Die Bauchlagerung in der Intensivmedizin ist auch für den Übergewichtigen eine Ultima Ratio in der Therapie des schweren ARDS, wenn der Organismus durch die schwere Hypoxämie vital bedroht ist. Sie darf nur am intubierten und maschinell beatmeten Patienten angewendet werden.

Die Lagerungstherapie des Übergewichtigen bleibt eine Herausforderung an Mensch und Material - sie gelingt nur mit einer großen Zahl an geschulten Helfern und mit Spezialausrüstung. 


\section{Literatur}

Baraka A, Moghrabi R, Yazigi A (1987) Unilateral pulmonary oede$\mathrm{ma} /$ atelectasis in the lateral decubitus position. Anaesthesia 42, 171-174

Baxi V, Budhakar S (2010) Anesthesia management of a morbidly obese patient in prone position for lumbar spine surgery. I Craniovertebr Junction Spine 1, 55-57

Bein T (1998) Patientenlagerung - Kinetische Therapie in der Intensivmedizin. Anaesthesist 47, 74-80

Benumof JL (2004) Obesity, sleep apnea, the airway and anesthesia. Curr Opin Anaesthesiol 17, 21-30

Boyce IR, Ness T, Castroman P, Gleysteen II (2003) A preliminary study of the optimal anesthesia positioning for the morbidly obese patient. Obes Surg 13, 4-9

Brodsky JB (2002) Positioning the morbidly obese patient for anesthesia. Obes Surg 12, 751-758

Brodsky JB, Lemmens HJM, Brock-Utne JG, Saidman LJ (2003) Anesthetic considerations for bariatric surgery: proper positioning is important for laryngoscopy. Anesth Analg 96, 1837-1846 [Letter to the editor]

Brodsky JB, Lemmens HJM, Bruck-Utne JG, Vierra M, Saidman LI (2002) Morbid obesity and tracheal intubation. Anesth Analg 94, 732-736

Burns SM, Egloff MB, Ryan B, Carpenter R, Burns JE (1994) Effect of body position on spontaneous respiratory rate and tidal volume in patients with obesity, abdominal distension and ascites. Am J Crit Care 3, 102-106

Chanimov M, Evron S Haitov Z, Stolero S, Cohen ML, Friedland M, Shul I, Bahar M (2010) Accidental venous and dural puncture during epidural analgesia in obese parturients (BMI > $40 \mathrm{~kg}$ / $\mathrm{m}^{2}$ ): three different body positions during insertion. I Clin Anesth 22, 614-618

Chergui K, Choukroun G, Meyer P, Caen D (2007) Prone-positioning for a morbidly obese patient with acute respiratory distress syndrome: an opportunity to explore intrinsic positive end-expiratory pressure-lower inflection point interdependence. Anesthesiology 106, 1237-1239

Collins JS, Lemmens HJM, Brodsky JB, Brock-Utne JG, Levitan RM (2004) Laryngoscopy and morbid obesity: a comparison of the „sniff“ and „ramped“ positions. Obes Surg 14, 1171-1175

Ezri T, Hazin V, Warters D, Szmuk P, Weinbroum AA (2003) The endotracheal tube moves more often in obese patients undergoing laparoscopy compared with open abdominal surgery. Anesth Analg 96, 278-282

Fishman AP (1981) Down with the good lung. N Engl I Med 304, 537538

Frat JP, Gissot V, Ragot S, Desachy A, Runge I, Lebert C, Robert R, Association des Reanimateurs du Centre-Ouest (ARCO) study group (2008) Impact of obesity in mechanically ventilated patients: a prospective study. Intensive Care Med 34,19911998

Futier E, Constantin JM, Pelosi P, Chanques G, Kwiatkowski F, Jaber S, Bazin JE (2010) Intraoperative recruitment maneuver reverses detrimental pneumoperitoneum-induced respiratory effects in healthy weight and obese patients undergoing laparoscopy. Anesthesiology 113, 1310-1319
Goldhill DR, Imhoff M, McLean B, Waldmann C (2007) Rotational bed therapy to prevent and treat respiratory complications: a review and meta-analysis. Am J Crit Care 16, 50-61

Gudmundsson G, Cerveny M, Shasby DM (1997) Spirometric values in obese individuals. Effects of body position. Am / Respir Crit Care Med 156, 998-999

Hakala K, Maasilta P, Sovijärvi AR (2000) Upright body position and weight loss improve respiratory mechanics and daytime oxygenation in obese patients with obstructive sleep apnoea. Clin Physiol 20, 50-55

Hamilton CL, Riley ET, Cohen SE (1997) Changes in the position of epidural catheters associated with patient movement. Anesthesiology $86,778-784$

Hamza I, Smida M, Benhamou D, Cohen SE (1995) Parturient's posture during epidural puncture affects the distance from skin to epidural space. I Clin Anesth 7, 1-4

Hurewitz AN, Susskind H, Harold WH (1985) Obesity alters regional ventilation in lateral decubitus position. I Appl Physiol 59, 774783

Itasaka Y, Miyazaki S, Ishikawa K, Togawa K (2000) The influence of sleep position and obesity on sleep apnea. Psychiatry Clin Neurosci 54, 340-341

Lewandowski K, Lewandowski M (2009) Lagerungstherapie des Patienten mit Adipositas per magna in der Intensivmedizin. In: Bein T (Hrsg.) Lagerungstherapie in der Intensivmedizin. 107136. MWV Medizinisch Wissenschaftliche Verlagsgesellschaft Berlin

Lopez PP, Stefan B, Schulman Cl, Byers PM (2008) Prevalence of sleep apnea in morbidly obese patients who presented for weight loss surgery evaluation: more evidence for routine screening for obstructive sleep apnea before weight loss surgery. Am Surg 74, 834-838

Meininger D, Zwissler B, Byhan C, Probst M, Westphal K, Bremerich DH (2006) Impact of overweight and pneumoperitoneum on hemodynamics and oxygenation during prolonged laparoscopic surgery. World I Surg 30, 520-526

Pankow W, Podszus T, Gutheil T, Penzel T, Peter J-H, von Wichert P (1998) Expiratory flow limitation and intrinsic positive end-expiratory pressure in obesity. I Appl Physiol 85, 1236-1243

Paul DR, Hoyt IL, Boutros AR (1976) Cardiovascular and respiratory changes in response to change of posture in the very obese. Anesthesiology 45, 73-78

Pelosi P, Croci M, Calappi E, Mulazzi D, Cerisara M, Vercesi P, Vicardi P, Gattinoni L (1996) Prone positioning improves pulmonary function in obese patients during general anesthesia. Anesth Analg 83, 578-583

Pelosi P, Ravagnan I, Giurati G, Panigada M, Bottino N, Tredici S, Eccher G, Gattinoni L (1999) Positive end-expiratory pressure improves respiratory function in obese but not in normal subjects during anesthesia and paralysis. Anesthesiology 91, 12211231

Perilli V, Sollazzi L, Bozza P, Modesti C, Chierichini A, Taccino RM, Ranieri $R$ (2000) The effects of the reverse Trendelenburg position on respiratory mechanics and blood gases in morbidly obese patients during bariatric surgery. Anesth Analg 91, 1520-1525

Perilli V, Sollazzi L, Modesti C, Annetta MG, Sacco T, Bocci MG, Tacchino RM, Proietti R (2003) Comparison of positive end-expiratory pressure with reverse Trendelenburg position in morbidly obese 
patients undergoing bariatric surgery: effects on hemodynamics and pulmonary gas exchange. Obes Surg 13, 605-609

Plywaczewski R, Bielen P, Bednarek M, Jonczak L, Gorecka D, Sliwinski $P$ (2008) Influence of neck circumference and body mass index on obstructive sleep apnea severity in males. Pneumonol Alergol Pol 76, 313-320

Protti A, Chiumello D, Cressoni M, Carlesso E, Mietto C, Berto V, Lazzerini M, Quintel M, Gattinoni L (2009) Relationship between gas exchange response to prone position and lung recruitability during respiratory failure. Intensive Care Med 35, 1011-1017

Rassam S, Sandbythomas M, Vaughan RS, Hall JE (2005) Airway management before, during and after extubation: a survey of practice in the United Kingdom and Ireland. Anaesthesia 60, 995-1001

Rheder K (1998) Postural changes in respiratory function. Acta Anaesthesiol Scand 113 (Suppl), 13-16

Sprung I, Whalley DG, Falcone T, Wilks W, Navratil JE, Bourke DL (2003) The effects of tidal volume and respiratory rate on oxygenation and respiratory mechanics during laparoscopy in morbidly obese patients. Anesth Analg 97, 268-274

Talab HF, Zabani IA, Abdelrahman HS, Bukhari WL, Mamoun I, Ashour MA, Sadeq BB, El Sayed SI (2009) Intraoperative ventilatory strategies for prevention of pulmonary atelectasis in obese patients undergoing laparoscopic bariatric surgery. Anesth Analg 109, 1511-1516
Tsueda K, Debrand M, Zeok SS, Wright BD, Griffin WO (1979) Obesity supine death syndrome: reports of two morbidly obese patients. Anesth Analg 58, 345-347

Valenza F, Vagginelli F, Tiby A, Francesconi S, Ronzoni G, Gugliemi M, Zappa M, Lattuada E, Gattinoni L (2007) Effects of the beach chair position, positive end-expiratory pressure, and pneumoperitoneum on respiratory function in morbidly obese patients during anesthesia and paralysis. Anesthesiology 107, 725-732

Vaughan RW, Wise L (1976) Intraoperative arterial oxygenation in obese patients. Ann Surg 184, 35-42

Vaughan RW, Wise L (1975) Postoperative arterial blood gas measurement in obese patients: Effect of position on gas exchange. Ann Surg 182, 705-709

Verse T, de la Chaux R, Dreher A, Fischer Y, Grundmann T, Hecksteden K, Hörmann K, Hohenhorst W, Ilgen F, Kühnel T, Mahl N, Maurer JT, Pirsig W, Roth B, Siegert R, Stuck BA (2008) Leitlinie: Therapie der obstruktiven Schlafapnoe des Erwachsenen. Laryngo Rhino Otol 87, 192-204

Watson RA, Pride NB (2005) Postural changes in lung volumes and respiratory resistance in subjects with obesity. I Appl Physiol 98, 512-517

Wyner J, Brodsky JB, Merell RC (1981) Massive obesity and arterial oxygenation. Anesth Analg 60, 691-693
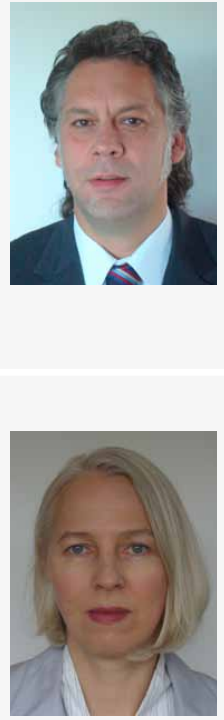

\section{Prof. Dr. med. Klaus Lewandowski}

Medizinstudium und Promotion an der Heinrich-Heine-Universität Düsseldorf. Habilitation und Professur am Rudolf-Virchow-Klinikum der Charité Berlin. Seit 2005 Direktor der Klinik für Anästhesiologie, Intensivmedizin und Schmerztherapie am Elisabeth-Krankenhaus Essen. Spezialgebiete in Klinik und Forschung: Epidemiologie und Intensivtherapie von Patienten mit ALI/ARDS, Anästhesie und Intensivtherapie von krankhaft übergewichtigen Patienten, Lungenprotektive maschinelle Ventilation, Exhaliertes Stickstoffmonoxid und $\mathrm{Na}$ sennebenhöhlen.

\section{Dr. med. Monika Lewandowski}

Medizinstudium und Promotion an der Heinrich-Heine-Universität Düsseldorf. Wissenschaftliche Mitarbeiterin im Fachbereich Anästhesiologie und operative Intensivmedizin an der Universitätsklinik Düsseldorf und der Universitätsklinik der Freien Universität Berlin. Danach Weiterbildungsstudium im Bereich Mathematik/Statistik/EDV an der Fernuniversität Hagen. Mitarbeit an wissenschaftlichen Forschungsprojekten mit den Schwerpunkten Exhaliertes Stickstoffmonoxid und Nasennebenhöhlen. In den letzten Jahren publizistische Tätigkeiten zu den Themen Maschinelle Ventilation, Stickstoffmonoxid und Anästhesie und Intensivtherapie von krankhaft übergewichtigen Patienten. 